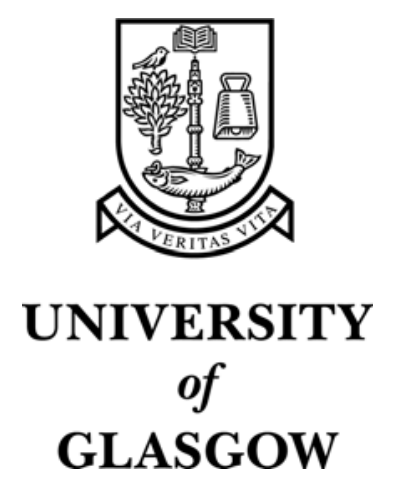

Wu, O. and Knill-Jones, R. and Wilson, P. and Craig, N. (2004) The impact of economic information on medical decision making in primary care. Journal of Evaluation in Clinical Practice 10(3):pp. 407-411.

http://eprints.gla.ac.uk/archive/00002850/ 
Journal of Evaluation in Clinical Practice, 10, 3, 407-411

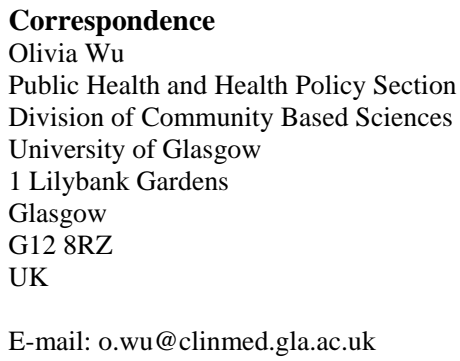

Keywords: decision making, medical economics, primary health care

Accepted for publication:

20 January 2004

\title{
The impact of economic information on medical decision making in primary care
}

\author{
Olivia Wu MSc, ${ }^{1}$ Robin Knill-Jones FFPH FRCP,, Philip Wilson DPhil MRCP FRCGP² and \\ Neil Craig MSc HonMFPH ${ }^{1}$
}

${ }^{1}$ Section of Public Health and Health Policy, Division of Community Based Sciences, University of Glasgow, UK

${ }^{2}$ Section of General Practice and Primary Care, Division of Community Based Sciences, University of Glasgow, UK

\begin{abstract}
Background Many general practitioners (GPs) are concerned about the increasing dominance of economic issues in major decisions about clinical care, and feel their opinions on economic matters have not been heard. It is unclear whether this information has any impact on everyday clinical practice in a primary care setting.

Aim To investigate GPs’ perspectives on the use of economic information in medical decision making. Design of study Cross-sectional survey.

Setting GP members of the West of Scotland Primary Care Research and Development Network (WestNet).

Methods Questionnaire survey sent to GPs by post and by email.

Results The overall response rate was $44 \%$, favouring postal over email responses. All respondents indicated that economic information has previously influenced them and should be incorporated into their medical decision making. The most common source of this information was generated by local authorities such as health boards, primary care groups and local prescribing advisors - used by $80 \%$ of the respondents. However, publications, such as the British Journal of General Practice, locally produced newsletters and prescribing formularies, and feedback from the General Practice Administration System for Scotland, were used as sources of economic information by $20 \%, 27 \%$ and $33 \%$, respectively. Published materials - in particular, locally specific information and summarized information in leaflet format - were favoured (54\%) in comparison to verbally presented material.
\end{abstract}

Conclusions GPs believe that economic information should be incorporated in medical decision making. The need for precise and summarized information, produced locally, has been highlighted. Better understanding towards the type of economic evidence GPs find useful and comprehensible is required. 


\section{Introduction}

The present health care culture demands proper consideration of the economic aspects of all health technologies. This has been highlighted by the introduction of groups such as National Institute for Clinical Excellence and National Health Service (NHS) Quality Improvement Scotland in the UK. Health economics is now a common term in public policy documents, scientific literature and even the lay press. 'Cost effectiveness' has become a major concern for health policy-makers, and economic evaluation is an integral part of drug development and health technology assessment. There is an increasing volume of economic literature about health care. These range from simple recommendations on cost issues to purposefully designed economic evaluations. However, it is unclear whether this information has any impact on everyday clinical practice in a primary care setting.

Few studies have explored the use of economic evaluation by decision makers in the UK (Drummond et al. 1997; Duthie et al. 1999; Hoffmann \& Graf von der Schulenburg 2000; Hoffmann et al. 2002). The most extensive survey was conducted by Drummond et al. (1997). Postal questionnaires were sent to prescribing advisers $(n=283)$, directors of pharmacy $(n=400)$ and directors of public health $(n=101)$, to evaluate several issues of economic evaluation: decision makers' knowledge of economics, the importance of efficacy as a decision-making criterion, the source of costs and outcomes information used by decision makers, the barriers to the use of economic evaluation and the use of the results of economic evaluations. The study concluded that the validity of economic studies and the inflexibility of budgets are the major obstacles to implementing the results from economic evaluations. These results were confirmed by those from the European Network on Methodology and Application of Economic Evaluation Techniques (EUROMET) project (Hoffmann et al. 2000), when decision makers $(n=1022)$ over nine European countries (including the UK) were surveyed by postal questionnaire, semi-structured interviews, or through focus groups.

Other surveys (Duthie et al. 1999; Hoffmann et al. 2002) have indicated a need for changes in conducting economic research. A qualitative survey on a group of mangers and clinicians $(n=34)$ found that results from economic evaluations were generally not well understood and were considered irrelevant (Duthie et al. 1999). A more recent study by Hoffmann et al. has drawn similar conclusions (Hoffmann et al. 2002). Based on a focus group from two UK health authorities (Leicestershire and North Yorkshire), the authors concluded that the generalizability, validity and quality of the economic studies are the major factors affecting implementation of economic information in clinical practice.

Many general practitioners (GPs) are concerned about the increasing dominance of economic issues in major decisions about clinical care, and feel their opinions on economic matters have not been heard. This is a short exploratory survey among members of the West of Scotland Primary Care Research and Development Network (WestNet). WestNet is a consortium of 55 general practices, set up to develop and conduct non-commercial research of benefit to general practice. The first aim of this study was to gain insight into GPs' views on the usefulness of a variety of economic information and how it related to their everyday practice. The second aim was to compare email and postal questionnaires response rates.

Methods Two questionnaires were designed and disseminated among GP members of WestNet. Questionnaire (I) consisted of five questions, designed to examine the type of economic information currently used by GPs in medical decision making. Questionnaire (II) consisted of four questions, designed to explore what GPs identify as sources of economic information and their perception on the relevance of the data presented.

A complete list of members and their contact details were obtained from WestNet. All the non-GP members were excluded from the study. Those included were randomized into two groups - questionnaire (I) and questionnaire (II), stratified by their accessibility by email. Each member was either sent the appropriate questionnaire with a personalized covering letter and a pre-paid addressed envelope, or emailed the covering letter, with the appropriate questionnaire attached.

Results A total of 53 members and 17 affiliated members were identified by the WestNet database. Four non- GP members (pharmacist, optometrist, clinical auditor and dentist) were excluded from the survey. All 
the eligible GPs ( $n=66)$ were divided into two groups - email group, that is, those with a contact email addresses $(n=33)$ and postal group, that is, those without contact email addresses $(n=33)$. However, email was undeliverable to five members - three from questionnaire (I) group and two from questionnaire (II) group.

\section{Response rates}

A total of 27 GPs returned the questionnaires, an overall response rate of $44 \%$. Table 1 gives a detailed breakdown of the response rate by each group. Higher response rates were observed with the postal group when compared with the email group and with questionnaire (I) when compared with (II). However, none of the differences between groups were significant.

Table 1 Survey response rates (number returned/number sent)

\begin{tabular}{|l|l|l|l|}
\hline & Questionnaire (I) & Questionnaire (II) & Total \\
\hline Email & $6 / 13(46 \%)$ & $4 / 15(27 \%)$ & $10 / 28(36 \%)$ \\
\hline Postal & $9 / 17(53 \%)$ & $8 / 16(50 \%)$ & $17 / 33(51 \%)$ \\
\hline Total & $15 / 30(50 \%)$ & $12 / 31(39 \%)$ & \\
\hline
\end{tabular}

Questionnaire (I) - economic information used in medical decision making

All respondents indicated they believe that economic information comparing cost and effectiveness of treatments has influenced their medical decision making. The majority of the respondents $(n=9 ; 69 \%)$ reported that such a decision was made as recently as 1 month previously or less, while $15 \%(n=2)$ reported such decisions made $1-6$ months ago and another 15\% $(n=2)$, over 6 months ago.

Both published and verbal economic information produced by local authorities (health boards, primary care groups, prescribing medical advisors and medical prescribing advisors) were the most commonly used by GPs, followed by information generated by the pharmaceutical industry (Table 2). However, only 13\% ( $n=$ $2)$ and $20 \%(n=3)$ have used conferences, seminars, and journal articles as economic information sources, respectively. A slight preference to published information was noted.

All respondents ( $n=15)$ uniformly reported changes in prescribing as their recent economic informationinfluenced medical decision. Change in proton pump inhibitors prescribing was described by 33\% $(n=5)$, statin prescribing in 20\% $(n=3)$, and 13\% $(n=2)$ described changes in the prescribing of non-steroidal anti-inflammatory drugs. Four respondents described circumstances when economic information had failed to influence their decision making. Fifty per cent $(n=2)$ felt it was 'impossible to implement these findings into practice', while one respondent disagreed with the results of the information presented. One respondent described the reason to be due to the 'cost of time in implementing changes not being reimbursed'.

Table 2 Economic information influencing decision making

\begin{tabular}{|l|l|}
\hline & 'Yes' n (\%) \\
\hline Published information & \\
\hline Local health board/PCG/PPAs/MPAs & $12(80 \%)$ \\
\hline Articles in journals & $3(20 \%)$ \\
\hline Industry literature & $5(33 \%)$ \\
\hline Others & $2(13 \%)$ \\
\hline Verbally presented information & \\
\hline Meetings with representatives from local health board/PCG/PPAs/MPAs & $6(40 \%)$ \\
\hline Conferences and seminars & $2(13 \%)$ \\
\hline Pharmaceutical industry representatives & $5(33 \%)$ \\
\hline Others & $2(13 \%)$ \\
\hline
\end{tabular}

PCG, primary care group; PPA, prescribing medical advisor; MPA, medical prescribing advisor.

Missing data $n=0$. 
Questionnaire (II) - sources and relevance of economic information

All respondents believed that economic information should be incorporated in health care decision making. They were all, with the exception of one $(n=11 ; 92 \%)$, able to describe the various sources of economic information they had used (Table 3). The most common source was the Scottish Prescribing Analysis (SPA) data - used by $83 \%$ of the respondents $(n=10), 80 \%(n=8)$ of whom found the material relevant to their everyday practice. This was followed by the literature produced by the pharmaceutical industry, which was used by $75 \%$ of the respondents $(n=9)$, but only $20 \%(n=2)$ found the material relevant to practice. Fifty-eight per cent $(n=7)$ recognized medical and pharmaceutical prescribing advisors as a source of economic information, while $62 \%(n=5)$ regarded the information as relevant.

However, 80\% $(n=8), 73 \%(n=8)$ and $67 \%(n=8)$ of the respondents did not regard the British Journal of General Practice, locally produced newsletters and prescribing formularies, and the General Practice Administration System for Scotland feedback as an economic information source.

Higher proportions of respondents preferred published material compared with verbally presented material (Table 4). In particular, locally specific information and summarized information in leaflet format were favoured by $54 \%(n=6)$ respondents.

Table 3 Sources of economic information used (numbers found the material relevant)

\begin{tabular}{|l|l|l|l|l|l|}
\hline & Yes & No & (Sometimes) & Don't know & Missing \\
\hline SPA prescribing feedback & $10(8)$ & $2(0)$ & $(2)$ & $0(0)$ & $0(2)$ \\
\hline Industry literature & $9(2)$ & $2(2)$ & $(4)$ & $1(2)$ & $0(2)$ \\
\hline MPAs and PPAs & $7(5)$ & $3(0)$ & $(2)$ & $2(1)$ & $0(4)$ \\
\hline British Medical Journal & $6(4)$ & $5(0)$ & $(2)$ & $0(0)$ & $1(6)$ \\
\hline Industry representatives & $6(1)$ & $5(2)$ & $(2)$ & $1(2)$ & $0(5)$ \\
\hline GPASS feedback & $4(2)$ & $8(0)$ & $(2)$ & $0(1)$ & $0(7)$ \\
\hline Local newsletter* & $3(3)$ & $8(1)$ & $(1)$ & $0(1)$ & $1(6)$ \\
\hline Other journals $\dagger$ & $3(2)$ & $3(0)$ & $(2)$ & $0(0)$ & $6(8)$ \\
\hline British Journal of General Practice & $2(1)$ & $8(0)$ & $(2)$ & $0(0)$ & $2(9)$ \\
\hline Local prescribing formulary & $2(1)$ & $8(0)$ & $(1)$ & $1(2)$ & $1(8)$ \\
\hline
\end{tabular}

SPA, Scottish Prescribing Analysis; MPA, medical prescribing advisor; PPA, prescribing medical advisor; GPASS, General Practice Administration System for Scotland.

*Newsletters generated from the Local Healthcare Co-operatives, Glasgow Prescriber.

†Prescriber, Journal of Health Economics, Bandolier, Drugs \& Therapeutics Bulletin.

Table 4 Preferred methods of presenting economic data

\begin{tabular}{|l|l|}
\hline & $\mathrm{n}(\%)$ \\
\hline Locally specific evaluations and studies & $6(54 \%)$ \\
\hline Summary of evaluations and studies published in leaflet format & $6(54 \%)$ \\
\hline Evaluations and studies published in literature & $5(45 \%)$ \\
\hline Simple recommendations presented in leaflet format & $5(45 \%)$ \\
\hline Summary of evaluations and studies presented verbally at a meeting & $3(27 \%)$ \\
\hline Simple recommendations presented verbally at a meeting & $1(9 \%)$ \\
\hline
\end{tabular}

Missing data $n=1$.

Discussion A GP perspective on economic information has not been explored previously. It is unclear what type of materials are being recognized and interpreted as economic information. The relevance and the extent of use of the wide variety of economic information being presented are also unknown.

Despite the small sample size and low response rate, this survey has provided some preliminary indication about GPs' perceptions and their use of economic information in medical decision making.

Although questionnaire (I) was designed to record recent activities - using economic information in decision making, while questionnaire (II) examined perceptions of economic information, the results were comparable. Both questionnaires showed the SPA data being recognized and used by most respondents as a source of economic information. The SPA data (level 1) consist of a breakdown of total costs and the number of items dispensed for major therapeutic areas. These are sent automatically to GPs, comparing 
their own average values with those of other practices, the health board and Scotland as a whole. However, such data contain solely cost information on prescribing and are not strictly an economic information source; they give no indication of the real quality of the prescribing taking place. Therefore, it is beneficial to consider including reliable economic information such as cost-effectiveness data, with the feedback information.

The results have also shown that published materials are used in preference to information from verbal presentations. In particular, locally specific evaluations and summary leaflets of studies were favoured by $54 \%$. However, $73 \%$ did not regard locally produced newsletters and prescribing formularies as economic sources. This may be an indication that locally produced newsletters and formularies lack adequate economic information that GPs find useful. The need for precise and summarized information, produced locally, has been highlighted.

Since the launch of the Scottish Office's primary care communications initiative in April 1997, 99\% of the practices in Scotland were computer-connected by the year 2000 (Willmot \& Sullivan 2000). However, a recent survey of internet connectivity and use in Lothian (Moffat et al. 2001) reported that $43 \%$ of their respondents spend no time using email. Therefore, it is not surprising that our survey has shown that email communications from GPs are still scarce and often unreliable.

It is clear that GPs recognize that economic information should be incorporated in medical decision making. However, the task of incorporating economic information into practice is a challenging one. Although all of our respondents in questionnaire (I) have indicated that economic information has previously influenced their medical decision making, four described situations where such information had failed to influence their decision making. This suggests that some economic information can only be applied in certain circumstances, and despite the effort and the cost spent on compiling and presenting such economic evidence, there is still wide variation in their usefulness and quality.

The results of the survey confirmed the difficulties of getting economics into practice. This revelation may not be novel, but it is important as it suggests that the message, as well as the information, is not getting through to GPs. Despite the time and money spent on compiling and synthesizing the evidence, and the prominent position GPs still occupy in the rationing process, especially after the creation of local health care co-operatives, there is little evidence on effective use of economic information. The study sample of this survey consisted of GPs who have a special interest in research. Although these GPs are not representative of all GPs in the UK, the results of this study are likely to be an underestimation of the prevalence of poor understanding and implementation of economic information in primary care.

\section{Acknowledgements}

The authors would like to thank all the GPs who participated in the survey.

\section{References}

Drummond M., Cooke J. \& Walley T. (1997) Economic evaluation under managed competition: evidence from the UK. Social Science \& Medicine 45, 583-595.

Duthie T., Trueman P., Chancellor J. \& Diez L. (1999) Research into the use of health economics in decision making in the United Kingdom - Phase II. Is health economics 'for good or evil'? Health Policy 46, 143-157.

Hoffmann C. \& Graf von der Schulenburg J.-M. (2000) The influence of economic evaluation studies on decision making: a European survey. Health Policy 52, 179-192.

Hoffmann C., Stoykova B.A., Nixon J., Glanville J.M., Misso K. \& Drummond M. (2002) Do health-care decision makers find economic evaluations useful? The findings of focus group research in UK health authorities. Value in Health 592, 71-79.

Moffat M.O., Moffat K.J. \& Cano V. (2001) General practitioners and the internet - a questionnaire survey of internet connectivity and use in Lothian. Health Bulletin 59 (2), 120-126.

Willmot M. \& Sullivan F. (2000) NHSnet in Scottish primary care. lessons for the future. British Medical Journal 321, 878-881. 